\title{
Theory and computation of higher gradient elasticity theories based on action principles
}

\begin{abstract}
In continuum mechanics, there exists a unique theory for elasticity, which includes the first gradient of displacement. The corresponding generalization of elasticity is referred to as strain gradient elasticity or higher gradient theories, where the second and higher gradients of displacement are involved. Unfortunately, there is a lack of consensus among scientists how to achieve the generalization. Various suggestions were made, in order to compare or even verify these, we need a generic computational tool. In this paper, we follow an unusual but quite convenient way of formulation based on action principles. First, in order to present its benefits, we start with the action principle leading to the well-known form of elasticity theory and present a variational formulation in order to obtain a weak form. Second, we generalize elasticity and point out, in which term the suggested formalism differs. By using the same approach, we obtain a weak form for strain gradient elasticity. The weak forms for elasticity and for strain gradient elasticity are solved numerically by using open-source packages-by using the finite element method in space and finite difference method in time. We present some applications from elasticity as well as strain gradient elasticity and simulate the so-called size effect.
\end{abstract}

\section{Introduction}

Starting with works Joseph-Louis de Lagrange, Pierre Louis Moreau de Maupertius, Leonhard Euler-for a short history see Romano et al. [38] - the theory of mechanics is formulated by an energy expression called LAGRANGEan that depends on primitive variables and their first derivatives in coordinates. Such a theory is of first order since the first derivative in coordinates describes the immediate neighborhood. This is an infinitesimally small region given by a sphere of radius $\varepsilon$. In continuum mechanics, we declare the size of $\varepsilon$ as approaching zero. Therefore, from a conceptual point of view, no problems arise and continuum mechanics is sufficient to characterize the immediate neighborhood with the first derivative. This approach is also called the locality argumentation and is valid for analytical mechanics, where we can set $\varepsilon$ infinitesimally small. However, if we want to exploit numerical mechanics, then we have to set a finite value for $\varepsilon$. It has to be smaller than the length-scale of the underlying structure.

B. E. Abali (《])

Department of Mechanical Engineering, University of California, Berkeley, 6124 Etcheverry Hall, Mailstop 1740, Berkeley, CA 94720 , USA

E-mail: b.e.abali@berkeley.edu

http://bilenemek.abali.org

W. H. Müller

Institute of Mechanics, Chair of Continuum Mechanics and Theory of Materials, Technische Universität Berlin, Einsteinufer 5, 10587 Berlin, Germany

F. dell'Isola

Dipartimento di Ingegneria Strutturale e Geotecnica, Sapienza Universita di Roma, Via Eudossiana 18, 00184 Roma, Italia 
In order to explain the length-scale, consider a measurement where the machine precision is limited to $1 \mu \mathrm{m}$. Therefore, we simply miss any deformation occurring on a smaller scale than a micrometer length-scale. We have to choose $\varepsilon$ smaller than $1 \mu \mathrm{m}$, and then we can accurately model the measurement. Moreover, we set $\varepsilon$ large enough such that sufficiently many atoms are included, so we may ignore any fluctuation effects. Suppose we can estimate $1 \mathrm{~nm}$ as the minimum size of $\varepsilon$, i.e., for a monatomic lattice we assume that first neighbor is in a distance less than $1 \mathrm{~nm}$. In a smaller length-scale, the atomistic structure and interactions between lattice points become important. Between $1 \mathrm{~nm}$ and $1 \mu \mathrm{m}$, the size of $\varepsilon$ can be chosen arbitrarily, and the solution of the problem remains the same; for the idea of self-similarity and intermediate distances, see Barenblatt [10, Chap. 2]. The first-order theory holds accurately for a material point in the given length-scale, i.e., $\varepsilon$ is smaller than the upper limit of $1 \mu \mathrm{m}$ and greater than the lower limit of $1 \mathrm{~nm}$. We can measure with a measurement device capturing $1 \mu \mathrm{m}$ and model the measurement with the first-order theory.

Now consider a material with $1 \mu \mathrm{m}$ grain size. In other words, the underlying material has a substructure within the length-scale. Now we have two different options to resolve this issue. Either we redeclare the lengthscale by decreasing the upper limit, simply stated, we buy a new measurement device with a higher precision; or we generalize the first-order theory in order to capture the effects of the substructure. This generalization introduces new coefficients responsible for the response effected by the substructure. In this work, we explain the method of least action and exploit it for generalizing the continuum theory from the first order to the second order.

Although the idea of an extended or generalized mechanics is older than a century, see dell'Isola et al. [14], the modern theory started to be developed by the early 1960s with Mindlin and Tiersten [29], Mindlin [27], Toupin [41], Eringen [18]. Today there are various theories describing an extension of the first-order theory for elasticity. Interestingly, these different theories are mathematically quite similar and can be viewed as specialized versions of a unified theory, see Neff et al. [31]. By starting with experimental evidence as in Morrison [30] and then later on in Yang and Lakes [43], Brezny and Green [12], Chen and Fleck [13], Kesler and Gibson [24], we know that a second-order theory—called the strain gradient theory-is necessary for an accurate prediction of experiments. For example, the prominent experiment of beam bending can be investigated. Consider a beam of $1 \mu \mathrm{m}$ length and another beam of $1 \mathrm{~mm}$, both out of the same material. The first-order theory is accurate for the beam in the meter length-scale; however, it is inaccurate for the beam in the micrometer length-scale. Apparently, for the micrometer beam, the substructure of the material becomes dominant. An internal substructure can be a crystallographic structure of aluminum or polymer chains of epoxy. If one wants to acquire accurate results with the first-order theory, then a precise and detailed model of this substructure needs to be involved. However, this method is not efficient at all. In other words, although we might try to model everything by molecular dynamics, we never do that for a structure bigger than a couple of micrometers due to the computational time required for such a detailed model. We will present a second-order theory, capable of modeling the deformation of beam bending even on smaller length-scales, with an adequate amount of computational cost.

Computational issues of generalized mechanics are heavily discussed in the literature. There are various propositions and numerical strategies. For example, Bilotta et al. [11] use a promising method by constructing special elements. The newly introduced material parameters can be motivated with a rigorous micro-macro identification procedure, see Pideri and Seppecher [33]. Based on this idea, Giorgio [19] provides a persuasive series of numerical results, starting from the first-order theory. In various applications, effects of substructure are unavoidable, for simulations of interesting examples, see Scerrato et al. [39], Turco et al. [42], and Placidi et al. [34]. Finite element implementation and comparable results to the underlying work have appeared recently in Reiher et al. [37]. We present our formulation and its implementation in a quite general setting; hence, an extension to many other branches than elasticity is possible. For example, the numerical solution procedure presented herein seems to be potentially very useful also for studying the equilibrium problems of second gradient fluids as discussed in Eremeyev [16], Eremeyev and Altenbach [17].

First, we discuss the variational formulation in the first-order theory by means of an action principle. Instead of the least action principle, another similar theory known as principle of virtual work (or power) can be used, too. We start by postulating an action and omit to give its detailed form. If a principle of virtual work needs to be used, then we immediately have to describe the form. There are no big discrepancies between these methods. Second, we generalize the variational formulation for the second-order theory by following Abali and Müller [3]. The outcome is a weak form necessary for the computation. Third, we present a numerical tool solving the weak form. For the case of elasticity and strain gradient elasticity, we perform several simulations in order to justify the benefits of the approach. We use open-source packages and make our codes publicly available in order to encourage further studies. 


\section{Variational formulation for the first-order theory}

The general formulation relies on a mathematical construct called action. The first postulate in this methodology is an existing LAGRANGEan density, $\mathcal{L}$, describing the underlying system

$$
\mathcal{L}=\mathcal{L}\left(x_{\mu}, \phi_{A}, \phi_{A, \mu}\right), \quad \phi_{A, \mu}=\frac{\partial \phi_{A}}{\partial x_{\mu}},
$$

which depends on Cartesian coordinates, $x_{\mu} \in \Omega, \mu=1,2, \ldots m$, in $m$-th-dimensional space, $\Omega \subset \mathbb{R}^{m}$; on primitive variables, $\phi_{A}=\phi_{A}\left(x_{\mu}\right)$; as well as on their derivatives, $\phi_{A, \mu}=\phi_{A, \mu}\left(x_{\mu}\right)$. Only the first derivatives are included in the list of arguments. The LAGRANGEan density describes the state of the underlying system. Thus, we call $\left\{\phi_{A}, \phi_{A, \mu}\right\}$ the state space. Consider a continuum body defined in the space $x_{\mu}=(t, x, y, z)$ is being deformed under a given mechanical loading. Then the state is the deformation of the body defined by the displacement field, $\phi_{A}=u_{i}=u_{i}(t, x, y, z)$, and its first gradient in coordinates. The space spanned by the displacement field and its first gradient in coordinates creates the state space. This concept is often used in terms of rigid bodies, where the displacement and velocity (derivative in $t$ ) create the state space.

The second postulate is the so-called action functional:

$$
\mathcal{A}=\int_{\Omega} \mathcal{L} \mathrm{d} \Sigma+\int_{\partial \Omega} W_{\mathrm{s}} \mathrm{d} \Gamma, \quad \mathrm{d} \Sigma=\mathrm{d} x_{1} \mathrm{~d} x_{2} \ldots \mathrm{d} x_{m}, \quad \mathrm{~d} \Gamma=\mathrm{d} x_{1} \mathrm{~d} x_{2} \ldots \mathrm{d} x_{m-1},
$$

where we have used the Cartesian coordinates such that the metric determinant of the space $\Omega$ is equal to 1 . If we call $\mathrm{d} \Omega$ an infinitesimal volume element, then one-dimension less $\mathrm{d} \Omega$ would be a surface element. We have a first-order theory such that only one-dimension lower space is included. Technically, we know $W_{\mathrm{s}}$ on the surface and want to determine the appropriate $\mathcal{L}$ within the space. Thus, $W_{\mathrm{s}}$ is given and we restrict it be depending on $\phi_{A}$. The action is a scalar, i.e., it is invariant with respect to the transformation of primitive variables. First, we introduce variations of the primitive variables,

$$
\phi_{A}^{\prime}=\phi_{A}+\varepsilon \delta \phi_{A},
$$

and of its rates,

$$
\phi_{A, \mu}^{\prime}=\phi_{A, \mu}+\varepsilon \delta \phi_{A, \mu}, \quad \delta \phi_{A, \mu}=\left(\delta \phi_{A}\right)_{, \mu}=\frac{\partial \delta \phi_{A}}{\partial x_{\mu}} .
$$

The test functions, $\delta \phi_{A}$, are arbitrary; however, they are chosen to vanish on the DIRICHLET boundaries, $\partial \Omega_{\mathrm{D}}$, where the primitive variables are known. The constant factor $\varepsilon$ is such a small number (with respect to the upper limit in the length-scale) that the above transformation is accurate in the first order in $\varepsilon$. Now, with the same accuracy, the variation of the action reads

$$
\delta \mathcal{A}=\int_{\Omega} \mathcal{L}^{\prime} \mathrm{d} \Sigma-\int_{\Omega} \mathcal{L} \mathrm{d} \Sigma+\int_{\partial \Omega} W_{\mathrm{s}}^{\prime} \mathrm{d} \Gamma-\int_{\partial \Omega} W_{\mathrm{s}} \mathrm{d} \Gamma, \quad \mathcal{L}^{\prime}=\mathcal{L}\left(x_{\mu}, \phi_{A}^{\prime}, \phi_{A, \mu}^{\prime}\right), \quad W_{\mathrm{s}}^{\prime}=W_{\mathrm{s}}\left(x_{\mu}, \phi_{A}^{\prime}\right) .
$$

By using a TAYLOR expansion linear in $\varepsilon$, we obtain

$$
\begin{aligned}
\mathcal{L}^{\prime}= & \mathcal{L}\left(x_{\mu}, \phi_{A}^{\prime}, \frac{\partial \phi_{A}^{\prime}}{\partial x_{\mu}}\right)=\mathcal{L}\left(x_{\mu}, \phi_{A}, \phi_{A, \mu}\right)+\frac{\partial \mathcal{L}\left(x_{\mu}, \phi_{A}, \phi_{A, \mu}\right)}{\partial \phi_{A}}\left(\phi_{A}^{\prime}-\phi_{A}\right) \\
& +\frac{\partial \mathcal{L}\left(x_{\mu}, \phi_{A}, \phi_{A, \mu}\right)}{\partial \phi_{A, \mu}}\left(\phi_{A, \mu}^{\prime}-\phi_{A, \mu}\right)=\mathcal{L}+\frac{\partial \mathcal{L}}{\partial \phi_{A}} \varepsilon \delta \phi_{A}+\frac{\partial \mathcal{L}}{\partial \phi_{A, \mu}} \varepsilon \delta \phi_{A, \mu},
\end{aligned}
$$

and analogously,

$$
W_{\mathrm{s}}^{\prime}=W_{\mathrm{s}}+\frac{\partial W_{\mathrm{s}}}{\partial \phi_{A}} \varepsilon \delta \phi_{A},
$$

where and henceforth over repeated indices we apply the EINSTEIN summation convention. The variation of action becomes

$$
\delta \mathcal{A}=\int_{\Omega}\left(\frac{\partial \mathcal{L}}{\partial \phi_{A}} \varepsilon \delta \phi_{A}+\frac{\partial \mathcal{L}}{\partial \phi_{A, \mu}} \varepsilon \delta \phi_{A, \mu}\right) \mathrm{d} \Sigma+\int_{\partial \Omega} \frac{\partial W_{\mathrm{s}}}{\partial \phi_{A}} \varepsilon \delta \phi_{A} \mathrm{~d} \Gamma
$$


According to the principle of least action, the action functional is a scalar such that its variation vanishes

$$
\delta \mathcal{A}=0,
$$

for non-dissipative (reversible) systems. Since $\varepsilon$ is a constant nonzero number, we can divide by it such that we obtain

$$
\int_{\Omega}\left(\frac{\partial \mathcal{L}}{\partial \phi_{A}} \delta \phi_{A}+\frac{\partial \mathcal{L}}{\partial \phi_{A, \mu}} \delta \phi_{A, \mu}\right) \mathrm{d} \Sigma+\int_{\partial \Omega} \frac{\partial W_{\mathrm{s}}}{\partial \phi_{A}} \delta \phi_{A} \mathrm{~d} \Gamma=0 .
$$

This integral form is general for a system having a LAGRANGEan density depending on the primitive variables and their first derivatives. Often, an integration by parts is established on the second term within $\Sigma$ such that the integrand contains only $\delta \phi_{A}$. This step introduces a surface integral derived from $\mathcal{L}$ set in with $\partial W_{\mathrm{s}} / \partial \phi_{A}$. Within $\Sigma$ the so-called EULER-LAGRANGE equations are obtained; over the surface, a boundary condition is defined. For enabling this relation, we have restricted $W_{\mathrm{s}}$ depending only on $\phi_{A}$ and not on $\phi_{A, \mu}$. Although we omit a direct use of this relation for generating the weak form, it serves an understanding of a boundary term and its specifications in an experimental setting.

\subsection{Generating the weak form}

Consider a material system composed of a three-dimensional continuum body in a reference frame, $\mathcal{B}_{0} \subset \mathbb{R}^{3}$, with its closure, $\partial \mathcal{B}_{0}$, such that $\overline{\mathcal{B}}=\mathcal{B}_{0} \cup \partial \mathcal{B}_{0}$. Material particles of the continuum body are denoted by their positions, $\boldsymbol{X}$, in the reference frame. The reference frame is an inertial frame, and we choose it as fixed. In the beginning of the simulation, where the continuum body is undeformed, we set the initial frame as the reference frame and indicate this choice with the use of an index " 0. ." Material particles' positions are expressed in a Cartesian coordinate system, $X_{i} \in \overline{\mathcal{B}}$ with $i=1,2,3$, in the initial frame, and they form an infinitesimal volume element $\mathrm{d} V=\mathrm{d} X_{1} \mathrm{~d} X_{2} \mathrm{~d} X_{3}$ as well as an infinitesimal surface element $\mathrm{d} A$. For this configuration, the space is four-dimensional, $x_{\mu}=\left(t, X_{i}\right)$, with time $t \in \tau=\left[t_{0}, t_{1}\right]$ and physical space $X_{i}$ such that the integral form in Eq. (10) reads

$$
\int_{\tau} \int_{\mathcal{B}_{0}}\left(\frac{\partial \mathcal{L}}{\partial \phi_{A}} \delta \phi_{A}+\frac{\partial \mathcal{L}}{\partial \phi_{A}^{*}} \delta \phi_{A}^{\cdot}+\frac{\partial \mathcal{L}}{\partial \phi_{A, i}} \delta \phi_{A, i}\right) \mathrm{d} t \mathrm{~d} V+\int_{\tau} \int_{\partial \mathcal{B}_{0}} \frac{\partial W_{\mathrm{s}}}{\partial \phi_{A}} \delta \phi_{A} \mathrm{~d} t \mathrm{~d} A=0,
$$

where $(\cdot)^{\cdot}$ denotes a partial time derivative since the reference frame is fixed. The integral form is continuous. In a computational environment, we will solve it for discrete time steps in discrete elements (subsets of the physical space). First, we discretize in time. For the sake of a simplified notation (and programming), we choose constant time steps such that the time becomes the following list:

$$
t=\left\{0, \Delta t, 2 \Delta t, 3 \Delta t, \ldots, t_{\mathrm{end}}\right\}
$$

As a consequence, the discrete (in time) representation of $\phi_{A}$ is a list,

$$
\phi_{A}=\left\{\phi_{A}\left(X_{i}, t=0\right), \phi_{A}\left(X_{i}, t=\Delta t\right), \phi_{A}\left(X_{i}, t=2 \Delta t\right), \ldots, \phi_{A}\left(X_{i}, t=t_{\mathrm{end}}\right)\right\} .
$$

The time rate of the primitive variables read

$$
\phi_{A}=\frac{\partial \phi_{A}}{\partial t}=\frac{\phi_{A}-\phi_{A}^{0}}{\Delta t},
$$

where $\phi_{A}$ denotes the unknown value to be computed and $\phi_{A}$ indicates the already computed value from the last time step. This formulation converges to the analytic time rate for $\Delta t \rightarrow 0$. Since the test functions $\delta \phi_{A}$ are arbitrary, we may set them as constants in the beginning of a simulation. For doing so, we have to eliminate $\delta \phi_{A}$ from the integral form by integrating by parts

$$
\begin{aligned}
& \int_{\tau} \int_{\mathcal{B}_{0}}\left(\frac{\partial \mathcal{L}}{\partial \phi_{A}} \delta \phi_{A}-\left(\frac{\partial \mathcal{L}}{\partial \phi_{A}^{*}}\right) \dot{\delta} \delta \phi_{A}+\frac{\partial \mathcal{L}}{\partial \phi_{A, i}} \delta \phi_{A, i}\right) \mathrm{d} V \mathrm{~d} t+\left.\int_{\mathcal{B}_{0}} \frac{\partial \mathcal{L}}{\partial \phi_{A}^{*}} \delta \phi_{A} \mathrm{~d} V\right|_{\tau} \\
& \quad+\int_{\tau} \int_{\partial \mathcal{B}_{0}} \frac{\partial W_{\mathrm{s}}}{\partial \phi_{A}} \delta \phi_{A} \mathrm{~d} t \mathrm{~d} A=0 .
\end{aligned}
$$


By given values of $\phi_{A}$ at two subsequent time instants like $t_{0}$ and $t_{1}$, we set the test functions zero on $\tau=\left[t_{0}, t_{1}\right]$ and obtain for every other time instant

$$
\int_{\mathcal{B}_{0}}\left(\frac{\partial \mathcal{L}}{\partial \phi_{A}} \delta \phi_{A}-\left(\frac{\partial \mathcal{L}}{\partial \phi_{A}^{*}}\right) \cdot \delta \phi_{A}+\frac{\partial \mathcal{L}}{\partial \phi_{A, i}} \delta \phi_{A, i}\right) \mathrm{d} V+\int_{\partial \mathcal{B}_{0}} \frac{\partial W_{\mathrm{s}}}{\partial \phi_{A}} \delta \phi_{A} \mathrm{~d} A=0
$$

Second, we discretize the physical space. The primitive variables as well as the test functions are defined on a HILBERT space with continuity $k$ in a discrete element, $E$, with the DIRICHLET boundary condition on $\partial E_{\mathrm{D}}$,

$$
\hat{\mathcal{V}}=\left\{\phi_{A}, \delta \phi_{A} \in\left[\mathcal{H}^{k}(E)\right]^{3}: \phi_{A}=\text { given, } \delta \phi_{A}=0 \text { on } \partial E_{\mathrm{D}}\right\}
$$

It is technically a SOBOLEV space where also the differentiability within the domain is ensured. The continuity $k$ shall be as high as necessary. It depends on the chosen LAGRANGEan density, which has a derivation in $\phi_{A, i}$. In the EULER-LAGRANGE equations, one more integration by parts is used to "switch" the derivative in the test function to that term. Hence, the regularity condition is stronger than the latter form. Therefore, the latter is called a weak form to be fulfilled in each element, $E$, finite in size. The totality of all finite elements reads the following weak form:

$$
\text { Form }=\sum_{n=1}^{\text {nr of ele. }} \int_{E^{n}}\left(\frac{\partial \mathcal{L}}{\partial \phi_{A}} \delta \phi_{A}-\left(\frac{\partial \mathcal{L}}{\partial \phi_{A}^{\cdot}}\right) \dot{\delta} \phi_{A}+\frac{\partial \mathcal{L}}{\partial \phi_{A, i}} \delta \phi_{A, i}\right) \mathrm{d} V+\int_{\partial \mathcal{B}_{0}} \frac{\partial W_{\mathrm{s}}}{\partial \phi_{A}} \delta \phi_{A} \mathrm{~d} A .
$$

The external work done on the surface is given, $W_{\mathrm{s}}=W_{\mathrm{s}}\left(t, X_{i}, \phi_{A}\right)$, and the surface element $\mathrm{d} A$ denotes an integration over the surface belonging to boundary $\partial \mathcal{B}_{0}$ excluding DIRICHLET boundaries. On the DIRICHLET boundaries, the numerical value of $\phi_{A}$ is given, since we know them $\delta \phi_{A}$ on the DIRICHLET boundaries vanishes, called GALERKIN approach. The weak form is a general integral form applicable to all reversible systems defined by a LAGRANGEan depending on primitive variables and their derivatives.

\subsection{Elasticity theory}

In elasticity the primitive variable is the displacement:

$$
\phi_{A}=u_{i}, \quad u_{i}=x_{i}-X_{i},
$$

where the displacement is the deviation from the reference (initial) position, $X_{i}$, to the current position, $x_{i}$. The LAGRANGEan density for the elasticity reads

$$
\mathcal{L}=\frac{1}{2} \rho_{0} u_{i} u_{i}-w+\rho_{0} f_{i} u_{i},
$$

where the first term denotes the kinetic energy, the second term indicates the stored or deformation energy, and the third term is called the potential energy. In the case of elasticity, the stored energy density (energy per volume) $w$ depends on displacement and its first derivative in space. Its exact form relies on the underlying material. The conservative force $f_{i}$ is simply the gravitational specific force (force per mass). We present two different material models in the following sections, and they only differ in the definition of the stored energy density, $w$. On NEUMANN boundaries, the energy expression

$$
W_{\mathrm{s}}=\hat{t}_{i} u_{i}
$$

implements a traction vector $\hat{t}_{i}$ in the unit of stress, modeling a mechanical loading on a part of the surface. By inserting the latter in Eq. (18), we obtain the weak form discrete in time and space,

$$
\text { Form }=\sum_{n=1}^{\text {nr of ele. }} \int_{E^{n}}\left(f_{i} \delta u_{i}-\rho_{0} \frac{u_{i}-2 u_{i}^{0}+u_{i}^{00}}{\Delta t \Delta t} \delta u_{i}-\frac{\partial w}{\partial u_{i, j}} \delta u_{i, j}\right) \mathrm{d} V+\int_{\partial \mathcal{B}_{0}} \hat{t}_{i} \delta u_{i} \mathrm{~d} A .
$$


This weak form can be solved by using $k=1$ since we have only first-order derivative in $u_{i}$. In other words, linear continuous standard elements will be used. Indeed, the latter weak form can be solved for various definitions of the stored energy density. It is of importance to note that the term:

$$
\frac{\partial w}{\partial u_{i, j}},
$$

is called PIOLA stress:

$$
P_{j i}=\frac{\partial w}{\partial F_{i j}}
$$

since the deformation gradient, $F_{j i}$, leads to

$$
F_{j i}=\frac{\partial x_{j}}{\partial X_{i}}=\delta_{j i}+u_{j, i}, \quad \frac{\partial w}{\partial u_{i, j}}=\frac{\partial w}{\partial F_{k l}} \frac{\partial F_{k l}}{\partial u_{i, j}}=P_{l k} \frac{\partial u_{k, l}}{\partial u_{i, j}}=P_{l k} \delta_{k i} \delta_{l j}=P_{j i} .
$$

The polynomial degree of Eq. (23) states the degree of the material model. The stored energy density is one degree higher, for example, for a linear material model $w$ is quadratic. By defining the LAGRANGEan density in Eq. (20), we have introduced several assumptions. First, we have assumed that it be independent of time. In other words, $f_{i}$ is a conservative force and does not depend on time explicitly. Second, we assume that the kinetic energy depends on $u_{i}^{*}$ and the stored energy depends on $u_{i}$ and $u_{i, j}$. In other words, we exclude viscoelasticity by neglecting a dependence on $u_{i, j}$. Third, we use the deformation gradient or the GREEN-LAGRANGE strain measure

$$
E_{i j}=\frac{1}{2} u_{k, i} u_{k, j}+u_{(i, j)},
$$

instead of displacement dependence in the stored energy. So the stored energy is

$$
w=\tilde{w}\left(X_{i}, F_{i j}\right)=\bar{w}\left(X_{i}, E_{i j}\right) .
$$

As a special case, for homogeneous materials the dependency on $X_{i}$ vanishes. Due to the principle of objectivity, the scalar function depends on the invariants of the deformation gradient or strain.

\section{Variational formulation for the second-order theory}

In order to generalize the proposed method, we redefine the LAGRANGEan density as including the second derivatives of the primitive variables

$$
\mathcal{L}=\mathcal{L}\left(x_{\mu}, \phi_{A}, \phi_{A, \mu}, \phi_{A, \mu \nu}\right), \quad \phi_{A, \mu}=\frac{\partial \phi_{A}}{\partial x_{\mu}}, \quad \phi_{A, \mu \nu}=\frac{\partial^{2} \phi_{A}}{\partial x_{\mu} \partial x_{\nu}} .
$$

In this configuration, the state space reads

$$
\left\{\phi_{A}, \phi_{A, \mu}, \phi_{A, \mu \nu}\right\}
$$

with the following variations:

$$
\phi_{A}^{\prime}=\phi_{A}+\varepsilon \delta \phi_{A}, \quad \phi_{A, \mu}^{\prime}=\phi_{A, \mu}+\varepsilon \delta \phi_{A, \mu}, \quad \phi_{A, \mu \nu}^{\prime}=\phi_{A, \mu \nu}+\varepsilon \delta \phi_{A, \mu \nu} .
$$

The same action definition and the variation of it lead to

$$
\mathcal{L}^{\prime}=\mathcal{L}+\frac{\partial \mathcal{L}}{\partial \phi_{A}} \varepsilon \delta \phi_{A}+\frac{\partial \mathcal{L}}{\partial \phi_{A, \mu}} \varepsilon \delta \phi_{A, \mu}+\frac{\partial \mathcal{L}}{\partial \phi_{A, \mu \nu}} \varepsilon \delta \phi_{A, \mu \nu},
$$

and provide the following integral form:

$$
\begin{aligned}
& \int_{\Omega}\left(\frac{\partial \mathcal{L}}{\partial \phi_{A}} \delta \phi_{A}+\frac{\partial \mathcal{L}}{\partial \phi_{A, \mu}} \delta \phi_{A, \mu}+\frac{\partial \mathcal{L}}{\partial \phi_{A, \mu \nu}} \delta \phi_{A, \mu \nu}\right) \mathrm{d} \Sigma+\int_{\partial \Omega} \frac{\partial W_{\mathrm{s}}}{\partial \phi_{A}} \delta \phi_{A} \mathrm{~d} \Gamma+\int_{\partial \Omega} \frac{\partial W_{\mathrm{s}}}{\partial \phi_{A, \mu}} \delta \phi_{A, \mu} \mathrm{d} \Gamma \\
& \quad+\int_{\partial \partial \Omega} \frac{\partial W_{\mathrm{e}}}{\partial \phi_{A}} \delta \phi_{A} \mathrm{~d} \Pi+\int_{\partial \partial \Omega} \frac{\partial W_{\mathrm{e}}}{\partial \phi_{A, \mu}} \delta \phi_{A, \mu} \mathrm{d} \Pi=0,
\end{aligned}
$$

after using the principle of least action and dividing by $\varepsilon$. By using the same justification as in the last section, we allow $W_{\mathrm{s}}$ on surface $\mathrm{d} \Gamma$ and $W_{\mathrm{e}}$ on edge $\mathrm{d} \Pi$ depending only on $\phi_{A}$ and $\phi_{A, \mu}$ but not on $\phi_{A, \mu \nu}$. 


\subsection{Generating the weak form}

As in the last section, again for $x_{\mu}=\left(t, X_{i}\right)$ with $t \in \tau=\left[t_{0}, t_{1}\right]$ and $X_{i} \in \mathcal{B}_{0}$, we obtain

$$
\begin{aligned}
& \int_{\tau} \int_{\mathcal{B}_{0}}\left(\frac{\partial \mathcal{L}}{\partial \phi_{A}} \delta \phi_{A}+\frac{\partial \mathcal{L}}{\partial \phi_{A}^{*}} \delta \phi_{A}^{\cdot}+\frac{\partial \mathcal{L}}{\partial \phi_{A, i}} \delta \phi_{A, i}+\frac{\partial \mathcal{L}}{\partial \phi_{A}^{*}} \delta \phi_{A}^{*}+\frac{\partial \mathcal{L}}{\partial \phi_{A, i}^{\cdot}} \delta \phi_{A, i}+\frac{\partial \mathcal{L}}{\partial \phi_{A, i j}} \delta \phi_{A, i j}\right) \mathrm{d} V \mathrm{~d} t \\
& +\int_{\tau} \int_{\partial \mathcal{B}_{0}} \frac{\partial W_{\mathrm{s}}}{\partial \phi_{A}} \delta \phi_{A} \mathrm{~d} t \mathrm{~d} A+\int_{\tau} \int_{\partial \mathcal{B}_{0}}\left(\frac{\partial W_{\mathrm{s}}}{\partial \phi_{A}^{*}} \delta \phi_{A}^{\cdot}+\frac{\partial W_{\mathrm{s}}}{\partial \phi_{A, i}} \delta \phi_{A, i}\right) \mathrm{d} t \mathrm{~d} A \\
& \quad+\int_{\tau} \int_{\partial \partial \mathcal{B}_{0}} \frac{\partial W_{\mathrm{e}}}{\partial \phi_{A}} \delta \phi_{A} \mathrm{~d} t \mathrm{~d} \ell+\int_{\tau} \int_{\partial \partial \mathcal{B}_{0}}\left(\frac{\partial W_{\mathrm{e}}}{\partial \phi_{A}^{*}} \delta \phi_{A}^{\cdot}+\frac{\partial W_{\mathrm{e}}}{\partial \phi_{A, i}} \delta \phi_{A, i}\right) \mathrm{d} t \mathrm{~d} \ell=0 .
\end{aligned}
$$

In the time discrete fashion, we eliminate terms with rates of test functions by integrating by parts. Then we use the fact that the values $\phi_{A}^{0}$ and $\phi_{A}^{00}$ are known in the beginning such that $\delta \phi_{A}$ vanishes. After discretization in space, we obtain the following weak form

$$
\begin{aligned}
\text { Form }= & \sum_{n=1}^{\text {nr of ele. }} \int_{E^{n}}\left(\frac{\partial \mathcal{L}}{\partial \phi_{A}} \delta \phi_{A}-\left(\frac{\partial \mathcal{L}}{\partial \phi_{A}^{*}}\right)^{\cdot} \delta \phi_{A}+\frac{\partial \mathcal{L}}{\partial \phi_{A, i}} \delta \phi_{A, i}+\left(\frac{\partial \mathcal{L}}{\partial \phi_{A}^{*}}\right)^{*} \delta \phi_{A}-\left(\frac{\partial \mathcal{L}}{\partial \phi_{A, i}^{*}}\right)^{\cdot} \delta \phi_{A, i}\right. \\
& \left.+\frac{\partial \mathcal{L}}{\partial \phi_{A, i j}} \delta \phi_{A, i j}\right) \mathrm{d} V++\int_{\partial \mathcal{B}_{0}}\left(\left(\frac{\partial W_{\mathrm{s}}}{\partial \phi_{A}}-\left(\frac{\partial W_{\mathrm{s}}}{\partial \phi_{A}^{*}}\right)^{\cdot}\right) \delta \phi_{A}+\frac{\partial W_{\mathrm{s}}}{\partial \phi_{A, i}} \delta \phi_{A, i}\right) \mathrm{d} A \\
& +\int_{\partial \partial \mathcal{B}_{0}}\left(\left(\frac{\partial W_{\mathrm{e}}}{\partial \phi_{A}}-\left(\frac{\partial W_{\mathrm{e}}}{\partial \phi_{A}^{*}}\right)\right)^{\cdot} \delta \phi_{A}+\frac{\partial W_{\mathrm{e}}}{\partial \phi_{A, i}} \delta \phi_{A, i}\right) \mathrm{d} \ell .
\end{aligned}
$$

The extension to the second-order theory introduced many different boundary conditions. All of them have to be given, and they are discussed by many researchers. Mainly we have boundary terms acting on surface and additionally terms acting on edges. Since now not only $\phi_{A}$ but also its derivative is involved, we need to approximate the discrete representation of $\phi_{A}$ from a SOBOLEV space,

$$
\hat{\mathcal{V}}=\left\{\phi_{A}, \delta \phi_{A} \in\left[\mathcal{H}^{k}(E)\right]^{3}: \phi_{A}=\text { given, } \delta \phi_{A}=0 \text { on } \partial E_{\mathrm{D}}\right\},
$$

with $k \geq 2$.

\subsection{Strain gradient elasticity theory}

In strain gradient elasticity, the primitive variable is the displacement:

$$
\phi_{A}=u_{i} .
$$

For being able to compare the elasticity and strain gradient elasticity, we implement exactly the same surface energy and neglect the edge energy,

$$
W_{\mathrm{s}}=\hat{t}_{i} u_{i}, \quad W_{\mathrm{e}}=0,
$$

where the traction vector $\hat{t}_{i}$ denotes again the mechanical load on the NEUMANN boundary. These boundary conditions are heavily discussed in the literature, and their application to experimental mechanics is very challenging. For some useful interpretations of these surface and edge energies and how they affect the solution, among others, we refer to Auffray et al. [8], Javili et al. [22], Steigmann and dell'Isola [40]. Although we obtained these additional terms on boundaries, they are challenging to justify. For the specific case of a quadratic energy, by using variational formulation in Mindlin [28], by using principle of virtual power in Polizzotto [35], it is shown that $W_{\mathrm{s}}$ and $W_{\mathrm{e}}$ may include relations leading to a dependency in $u_{i}$ and $u_{i, j}$. Moreover, the aforementioned relations can be rewritten, see [35, Sect.5], in order to relate them to measurable quantities like surface tension (energy). Such an analysis results in $W_{\mathrm{s}}$ depending on $u_{i}$ and surface normal component of gradient of displacement. Herein, we have even proposed to involve dependencies on $u_{i}$. Unfortunately, we lack a clear justification of experimental feasibility for such dependencies. For simplicity, we choose Eq. (37) to implement such that a comparison with the well-known linear elasticity is possible. 
For nonpolar materials, we propose the following LAGRANGEan density:

$$
\mathcal{L}=\frac{1}{2} \rho_{0} u_{i} u_{i}-w+\rho_{0}\left(f_{i} u_{i}+l_{i j} u_{j, i}\right) .
$$

The first term is an inertial term. Herein, we simplify the formulation by assuming that only the velocity and ordinary momentum (mass times velocity) take part in this term, see Polizzotto [35], Polizzotto [36] for an elaborate discussion. So the inertial term consists of the kinetic energy due to the macroscopic velocity for nonpolar materials. For a polar material, this term would have a term with spin, too. The second term is again the stored energy; however, the list of argumentation is amended such that the stored energy depends not only on the first but also on the second gradient of the primitive variable, i.e., displacement,

$$
w=w\left(X_{i}, E_{i j}, E_{i j, k}\right) .
$$

We will restrict the formulation to homogeneous materials such that the dependency on material particles' positions, $X_{i}$, vanishes. The third term denotes the energy due to the volumetric effects. The first part, $f_{i} u_{i}$, is the specific energy because of the gravitational forces creating a translational displacement (implied by the linear momentum). The second part, $l_{i j} u_{j, i}$, is the specific volumetric energy leading to a rotational displacement (implied by the angular momentum). For a nonpolar medium, this term vanishes. By inserting the LAGRANGEan density, we obtain the weak form for a nonpolar material with traction-type boundaries:

$$
\begin{aligned}
\text { Form }= & \sum_{n=1}^{\text {nr of ele. }} \int_{E^{n}}\left(\rho_{0} f_{i} \delta u_{i}-\rho_{0} \frac{u_{i}-2 u_{i}^{0}+u_{i}^{00}}{\Delta t \Delta t} \delta u_{i}-\frac{\partial w}{\partial u_{i, j}} \delta u_{i, j}+\rho_{0} l_{j i} \delta u_{i, j}\right. \\
& \left.\quad-\frac{1}{\Delta t \Delta t}\left(\frac{\partial w}{\partial u_{i, j}}-2 \frac{\partial w^{0}}{\partial u_{i, j}^{0}}+\frac{\partial w^{00}}{\partial u_{i, j}^{00}}\right) \delta u_{i, j}-\frac{\partial w}{\partial u_{i, j k}} \delta u_{i, j k}\right) \mathrm{d} V+\int_{\partial \mathcal{B}_{0}} \hat{t}_{i} \delta u_{i} \mathrm{~d} A,
\end{aligned}
$$

where the following notation is introduced

$$
\begin{aligned}
& w^{0}=w\left(E_{i j}^{0}, E_{i j, k}^{0}\right), \quad w^{00}=w\left(E_{i j}^{00}, E_{i j, k}^{00}\right), \\
& E_{i j}^{0}=\frac{1}{2} u_{k, i}^{0} u_{k, j}^{0}+u_{(i, j)}^{0}, \quad E_{i j}^{00}=\frac{1}{2} u_{k, i}^{00} u_{k, j}^{00}+u_{(i, j)}^{00},
\end{aligned}
$$

with the GREEN-LAGRANGE strain tensor, $E_{i j}$, and its values in the last and second last time steps, $E_{i j}^{0}, E_{i j}^{00}$, respectively. The latter form can be solved by using $k=2$ since we have second-order derivative in $u_{i}$. In other words, we will use quadratic continuous standard elements.

\section{Applications}

The weak forms in Eq. (22) for elasticity and in Eq. (40) for strain gradient elasticity are general forms applicable to linear as well as nonlinear materials. Moreover, since we use a nonlinear strain measure, namely the GREENLAGRANGE strain tensor, the formulation captures geometric nonlinearities as well. Other than elastic response, we have not made any assumptions so far. In this section, we will present a computational tool for solving the presented nonlinear weak forms numerically. By using the Python programming language, see Oliphant [32], we develop a code for obtaining solution of weak forms by exploiting the collection of open-source packages developed under the FEniCS project, see Hoffman et al. [21], Logg et al. [26], Jones et al. [23]. We use finite element method in space and finite difference method in time. For the visualization of results, we use ParaView, see Ahrens et al. [5], Ayachit [9].

Three examples are shown for linear elastic, hyperelastic, and linear strain gradient elastic materials. All examples have nonlinear weak forms such that the weak form needs to be linearized before solving. This linearization is gained by a symbolic derivation of the weak form, before assembly. Therefore, the linearization is at the level of the partial differential equations. The derivation is fully automatized by using symbolic derivative functionality, see Alnæs and Mardal [6], Alnæs and Mardal [7]. Moreover, we have implemented the code by defining the stored energy function. Its derivation, precisely the solution of Eq. (23), is computed by using the aforementioned symbolic derivative functionality. This abstraction is of paramount importance, and the following three examples differ only in the definition of the stored energy function.

For learning the basics of programming in Python for FEniCS ecosystem, see Langanten and Logg [25]. For several engineering applications by using FEniCS, see Abali [1]. In order to encourage further research, we publish all used codes in Abali [2] under the GNU Public license as stated in Gnu Public [20]. 


\subsection{Example for linear elasticity}

A structure made of an engineering steel can be modeled with a linear elastic material if the mechanical loading generates stresses less than the yield stress such that no plastic deformation occurs. Consider a thin plate modeled as a three-dimensional structure under a mechanical loading. Under a concentrated loading, plate underlies a large deformation (with respect to the geometric dimensions). For the linear model of a homogeneous steel, the generic form of the quadratic scalar function reads

$$
w=E_{i j} C_{i j k l} E_{k l},
$$

where $C_{i j k l}$ is called the stiffness or elasticity tensor with 21 independent coefficients since

$$
C_{i j k l}=C_{j i k l}, \quad C_{i j k l}=C_{i j l k}, \quad C_{i j k l}=C_{k l i j},
$$

effected by the symmetric strain, $E_{i j}=E_{j i}$. This case is applicable for any material class. Existing crystal symmetries decrease the number of independent coefficients. For example, in the case of an isotropic material, the representation of this rank four tensor simplifies to

$$
C_{i j k l}=\lambda \delta_{i j} \delta_{k l}+\mu_{1} \delta_{i k} \delta_{j l}+\mu_{2} \delta_{i l} \delta_{j k},
$$

see, for example, Abali et al. [4, Appendix] for a derivation. By inserting the stiffness tensor into the energy density, we obtain the so-called ST. VENANT-KIRCHHOFF model for the stored energy:

$$
w=\lambda \frac{1}{2} E_{i i} E_{j j}+\mu E_{i j} E_{j i},
$$

where $\mu=\mu_{1}=\mu_{2}$ since strain is symmetric and the so-called LAME parameters, $\lambda, \mu$, are given by YounG's modulus, $E$, and PoISSON's ratio, $v$ as follows

$$
\lambda=\frac{E v}{(1+v)(1-2 v)}, \quad \mu=\frac{E}{2(1+v)} .
$$

The strain measure is nonlinear; thus, the geometric nonlinearities are computed accurately. The stored energy density is quadratic in strain; therefore, the material model in Eq. (23) is linear. We simulate a plate of an AISI steel with the following parameters:

$$
\rho_{0}=8.1 \times 10^{-9} \mathrm{Mg} / \mathrm{mm}^{3}, \quad E=200 \times 10^{3} \mathrm{MPa}, \quad v=0.3 .
$$

The plate is of $1000 \times 600 \times 20 \mathrm{~mm}$, and its surface lies in $X_{1} X_{2}$-plane. The concentrated force is modeled by a GAUSSian distribution, increasing linearly in time

$$
\tilde{t}=a t \exp \left(b\left(X_{1}-\bar{X}_{1}\right)^{2}+b\left(X_{2}-\bar{X}_{2}\right)^{2}\right),
$$

such that $\hat{t}_{i}=-\tilde{t} N_{i}$ applies on a small area on top of the plate against the plane normal, $N_{i}$. The force increases linearly in time. The gravitational forces are implemented, $f_{i}=(0,0,-9850) \mathrm{N} / \mathrm{Mg}$, although the deformation due to them is negligible (small with respect to the deformation caused by the loading). The transient solution of the weak form in Eq. (22) provides the displacement field, $u\left(t, X_{i}\right)$, as expected, for the convergence see Appendix A. The response is immediate, and the deformation goes back to the initial value after unloading. For example, the deformation is shown in Fig. 1 at the time instant of maximum force applied on the top of the plate. 


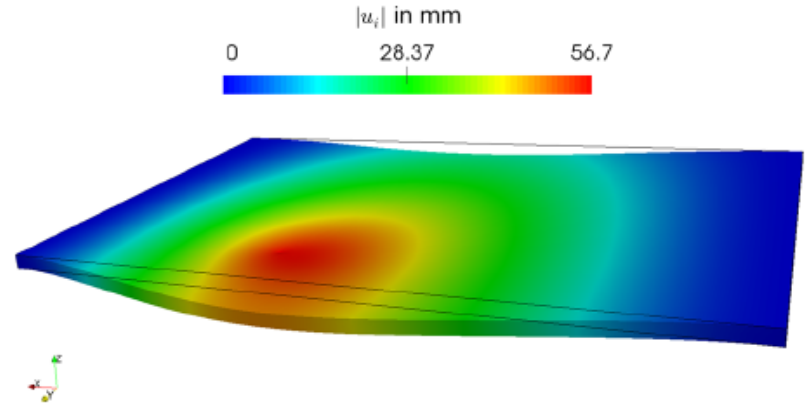

Fig. 1 Deformation of the three-dimensional elastic plate, shown without scaling; colors denote the magnitude of the displacement field, $\left|u_{i}\right|$

\subsection{Example for nonlinear elasticity}

A soft material, for example a silicon gel, has a more localized strain distribution leading to sharp strain alterations in the structure. A nonlinear material model is necessary to capture this effect. For a silicon gel, we implement the neo-HoOKEan energy density:

$$
w=\lambda \frac{1}{2} \ln ^{2}(\operatorname{det}(\boldsymbol{F}))+\mu\left(\frac{F_{j i} F_{j i}}{2}-\frac{\delta_{j j}}{2}-\ln (\operatorname{det}(\boldsymbol{F}))\right),
$$

where the deformation gradient occurs in a highly nonlinear fashion such that its derivative, the stress tensor, is nonlinear, too. The material parameters for the silicon gel TSE3062 are as follows

$$
\rho_{0}=1.1 \times 10^{-9} \mathrm{Mg} / \mathrm{mm}^{3}, \quad v=0.4, \quad E=89 \mathrm{MPa} .
$$

Since taking derivative of the energy is handled automatically, the implementation is the same as in the previous example, only the stored energy definition changes. We solve the silicon gel filled in a cup under a line loading by using the following GAUSSian distribution:

$$
\tilde{t}=a t \exp \left(b\left(X_{2}-\bar{X}_{2}\right)^{2}\right) .
$$

Since the deformation in a soft matter is much greater than the gravitational-based deflection, we assume $f_{i}=0$. The computation is as expected, for the convergence see Appendix B. The deformation at the time when the force reaches its maximum value is shown in Fig. 2.

\subsection{Example for linear strain gradient elasticity}

In order to generalize the stored energy formulation, we start with a generic representation for linear homogeneous materials:

$$
w=w\left(E_{i j}, E_{i j, k}\right)=E_{i j} C_{i j k l} E_{k l}+E_{i j, k} D_{i j k l m n} E_{l m, n}+E_{i j} G_{i j k l m} E_{k l, m} .
$$

For isotropic materials, the mixed term, $G_{i j k l m}$, vanishes, see dell'Isola et al. [15]. For an isotropic linear material, we obtain

$$
\begin{aligned}
C_{i j k l}= & \bar{c}_{01} \delta_{i j} \delta_{k l}+\bar{c}_{02} \delta_{i k} \delta_{j l}+\bar{c}_{03} \delta_{i l} \delta_{j k}, \\
D_{i j k l m n}= & c_{01} \delta_{i j} \delta_{k l} \delta_{m n}+c_{02} \delta_{i j} \delta_{k m} \delta_{l n}+c_{03} \delta_{i j} \delta_{k n} \delta_{m l}+c_{04} \delta_{i k} \delta_{j l} \delta_{m n} \\
& +c_{05} \delta_{i k} \delta_{j m} \delta_{l n}+c_{06} \delta_{i k} \delta_{j n} \delta_{l m}+c_{07} \delta_{i l} \delta_{j k} \delta_{m n}+c_{08} \delta_{i l} \delta_{j m} \delta_{k n} \\
& +c_{09} \delta_{i l} \delta_{j n} \delta_{m k}+c_{10} \delta_{i m} \delta_{j k} \delta_{l n}+c_{11} \delta_{i m} \delta_{j l} \delta_{k n}+c_{12} \delta_{i m} \delta_{j n} \delta_{l k} \\
& +c_{13} \delta_{i n} \delta_{j k} \delta_{l m}+c_{14} \delta_{i n} \delta_{j l} \delta_{k m}+c_{15} \delta_{i n} \delta_{j m} \delta_{k l},
\end{aligned}
$$

see Abali et al. [4, Appendix] for a derivation of these forms. From the quadratic energy, we realize the following conditions:

$$
C_{i j k l}=C_{k l i j}, \quad D_{i j k l m n}=D_{l m n i j k} .
$$




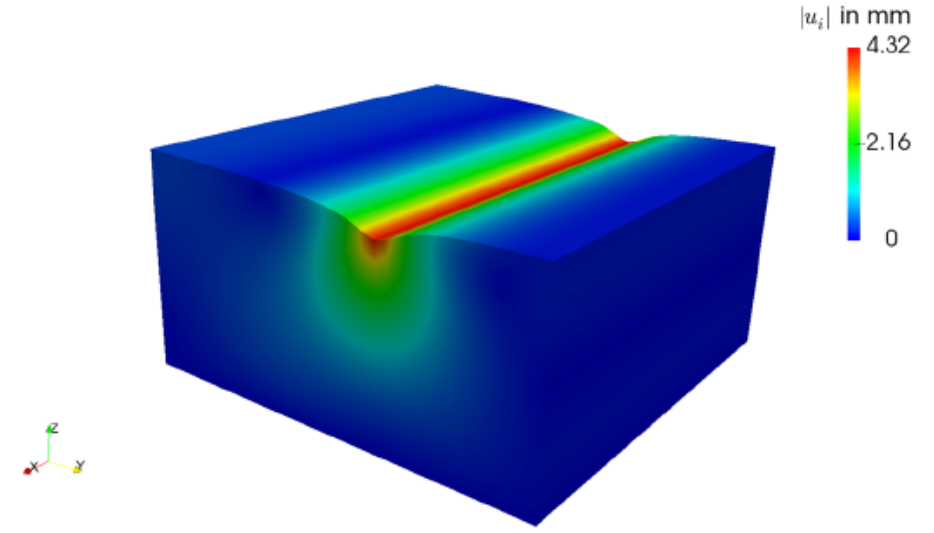

Fig. 2 Deformation of the silicon gel filled in a box of $80 \times 80 \times 40 \mathrm{~mm}$, presented without any scaling; colors denote the magnitude of the displacement field, $\left|u_{i}\right|$

Moreover, the symmetry in strains, $E_{i j}=E_{j i}$, implies further conditions:

$$
C_{i j k l}=C_{j i k l}, \quad C_{i j k l}=C_{i j l k}, \quad D_{i j k l m n}=D_{j i k l m n}, \quad D_{i j k l m n}=D_{i j k m l n} .
$$

By applying all of these conditions, we obtain

$$
\begin{aligned}
C_{i j k l}= & c_{1} \delta_{i j} \delta_{k l}+c_{2}\left(\delta_{i k} \delta_{j l}+\delta_{i l} \delta_{j k}\right), \\
D_{i j k l m n}= & c_{3}\left(\delta_{i j} \delta_{k l} \delta_{m n}+\delta_{i n} \delta_{j k} \delta_{l m}+\delta_{i j} \delta_{k m} \delta_{l n}+\delta_{i k} \delta_{j n} \delta_{l m}\right)+c_{4} \delta_{i j} \delta_{k n} \delta_{m l} \\
& +c_{5}\left(\delta_{i k} \delta_{j l} \delta_{m n}+\delta_{i m} \delta_{j k} \delta_{l n}+\delta_{i k} \delta_{j m} \delta_{l n}+\delta_{i l} \delta_{j k} \delta_{m n}\right)+c_{6}\left(\delta_{i l} \delta_{j m} \delta_{k n}+\delta_{i m} \delta_{j l} \delta_{k n}\right) \\
& +c_{7}\left(\delta_{i l} \delta_{j n} \delta_{m k}+\delta_{i m} \delta_{j n} \delta_{l k}+\delta_{i n} \delta_{j l} \delta_{k m}+\delta_{i n} \delta_{j m} \delta_{k l}\right) .
\end{aligned}
$$

Hence, for an isotropic linear homogeneous material including strain gradients (of order one), the generic energy formulation consists of seven material parameters.

The five newly introduced parameters, viz., $c_{3}, c_{4}, c_{5}, c_{6}$, and $c_{7}$, change the deformation of the structure. We have been able to detect one parameter, $c_{6}$, imposing the so-called size effect in a beam bending. In order to demonstrate how the proposed material model simulates the size effect in a beam bending, we construct a beam of length $\ell$. It is a slender beam, $\ell \times \ell / 30 \times \ell / 30$, out of aluminum with the following properties:

$$
\begin{aligned}
& \rho_{0}=2.7 \times 10^{-9} \mathrm{Mg} / \mathrm{mm}^{3}, \quad v=0.33, \quad E=72 \times 10^{3} \mathrm{MPa}, \\
& c_{1}=\lambda=\frac{E v}{(1+v)(1-2 v)}, \quad c_{2}=\mu=\frac{E}{2(1+v)} .
\end{aligned}
$$

By clamping one end at $x=0$ and shearing the other end at $x=\ell$, we simulate transiently the displacement in three-dimensional beam structure for various parameters. The solution is as expected, for the convergence see Appendix C. The shear force is applied on the whole cross section at $x=\ell$, and it increases linearly in $1 \mathrm{~s}$ up to $10 \mathrm{~N}$ for a beam of length $\ell=100 \mathrm{~mm}$. We set $c_{3}=c_{4}=c_{5}=c_{7}=0$ and vary $c_{6}$ parameter. In Table 1, the role of $c_{6}$ is studied. We recall that for a 100 -mm-long beam such displacements are considered as large deformations. We refrain ourselves from verifying the results to any analytic solution applicable to geometrically linear cases. The parameter $c_{6}$ causes a stiffness in the shear bending. By setting $c_{6}=0$, we obtain the results for elasticity. Interestingly, according to the introduction of the length-scale $\sqrt{c_{6} / E}$, see for a discussion of the length-scale in Giorgio [19], this particular effect depends on the size of the beam. In order to present this feature, we simulate the same beam of $\ell \times \ell / 30 \times \ell / 30$ in three different lengths: $\ell=10 \mathrm{~m}$, $\ell=10 \mathrm{~mm}$, and $\ell=10 \mu \mathrm{m}$. All material parameters are identical for these simulations with $c_{6}=1000 \mathrm{~N}$. Since the lengths are different, the deformation is different quantitatively. In order to obtain the same amount of deformation per length, we can match the shearing force by applying the same force per beam length square. This ratio should be held constant. As a reasoning consider the limiting case, where the force is so small that there occurs a geometrically linear deformation. The maximum deflection per beam length is proportional to

$$
u_{\max }=\frac{F \ell^{3}}{3 E I}, \quad I=\frac{\ell^{4}}{12 \cdot 30^{4}} \Rightarrow \frac{u_{\max }}{\ell} \propto \frac{F}{\ell^{2}} .
$$


Table 1 Maximum deflection

\begin{tabular}{lc}
\hline$c_{6}$ in N & $u_{i}^{\text {max. }}$ in mm \\
\hline 0 & 4.21286 \\
1 & 4.21285 \\
10 & 4.21274 \\
100 & 4.21164 \\
1000 & 4.20077 \\
\hline
\end{tabular}
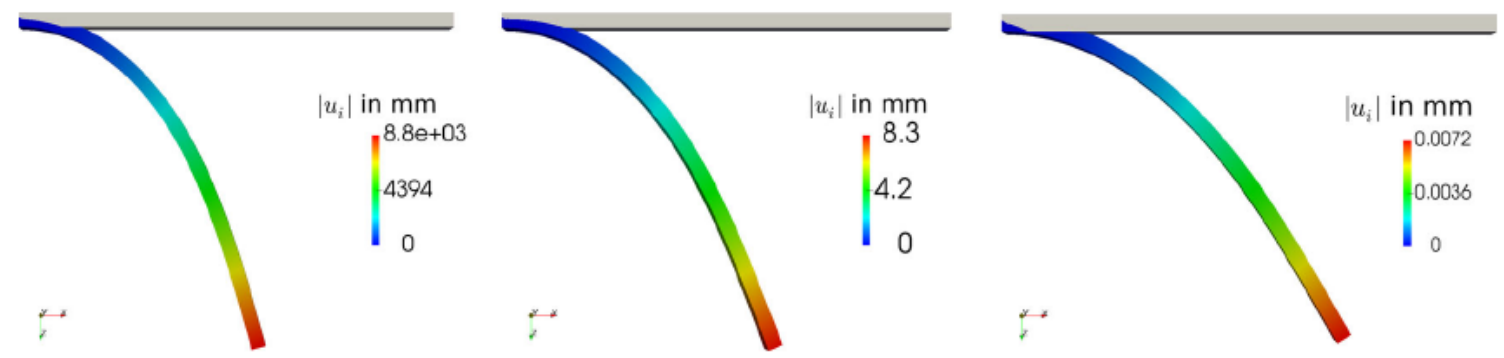

Fig. 3 Deformation of the three-dimensional cantilever beam bending with $c_{6}=1000 \mathrm{~N}$, without any scaling; colors denote the magnitude of the displacement field, $\left|u_{i}\right|$. Left $10-\mathrm{m}$ beam. Middle $10-\mathrm{mm}$ beam. Right $10 \mu \mathrm{m}$ beam

By choosing a large $F$ value and holding its ratio per beam length square fixed, we observe in Fig. 3 that the qualitative deformation changes depending on the beam length, and shorter beams present more stiffening. This stiffening effect is observed in experiments exactly as simulated herein. Often, this phenomenon is called size effect indicating that the deformation behavior changes depending on the size of the structure. Exactly this phenomenon is a possible procedure of determining the newly introduced $c_{6}$ parameter. A measurement of the tip deflection of beams in different length-scales is compared to computations with a particular $c_{6}$ parameter. This determined $c_{6}$ parameter can be used for structures larger than $\sqrt{c_{6} / E}$. For example, suppose that we have a material with $c_{6}=10 \mathrm{~N}$ and $E=72000 \mathrm{MPa}$, in this case $\sqrt{c_{6} / E}=11.79 \mu \mathrm{m}$ can be interpreted as the upper limit of the characteristic length, and in the beam bending this length is the beam length. A beam shorter than the upper limit, the substructure becomes relevant such that we have to model it in a detailed matter. In the numerical examples above, we have ignored this fact and devoted ourselves to interpreting the effect of the newly introduced parameter.

In order to present the reason why this stiffening effect emerges, we perform different simulations for a beam of $\ell=10 \mu \mathrm{m}$ with $c_{6}=0$ and $c_{6}=1000 \mathrm{~N}$. All deformations are plotted on top of each other in Fig. 4 . Although the shearing force is acting on the whole cross section, there is a skew bending introduced with $c_{6} \neq 0$. In order to preserve the positive definiteness of the stored energy, we choose $c_{6}$ positive, see dell'Isola et al. [15, Sect. 4]. We interpret the results as if an additional rotation such as a twist is implied. Instead of a pure shear bending, there is a shear bending and a torsion on $x_{1}$-axis (positively) due to $c_{6}$ parameter bringing in the effect of the internal substructure.

\section{Conclusion}

We have presented a general theory of generalized elasticity by means of LAGRANGEan mechanics by using a variational formulation. This method leads naturally to a weak form, which is an important benefit of using this strategy. By obtaining the weak form, we can implement a numerical computation of any physical system. In this work, we demonstrated the variational formulation in elasticity as well as in strain gradient elasticity theory. For the sake of presenting a general approach, we have left the stored energy density undefined. In order to simulate concrete examples, we defined a stored energy density for linear and nonlinear elasticity, as well as for linear strain gradient elasticity. By using finite element method in space and finite difference method in time, we implemented and solved concrete applications.

Although formulations for generating the weak form for elasticity exist in the literature, they start by postulating the balance of the linear momentum and then defining the stress tensor. In our formulation, we postulate the LAGRANGEan density and define the stored energy density. For the linear and nonlinear elasticity, 


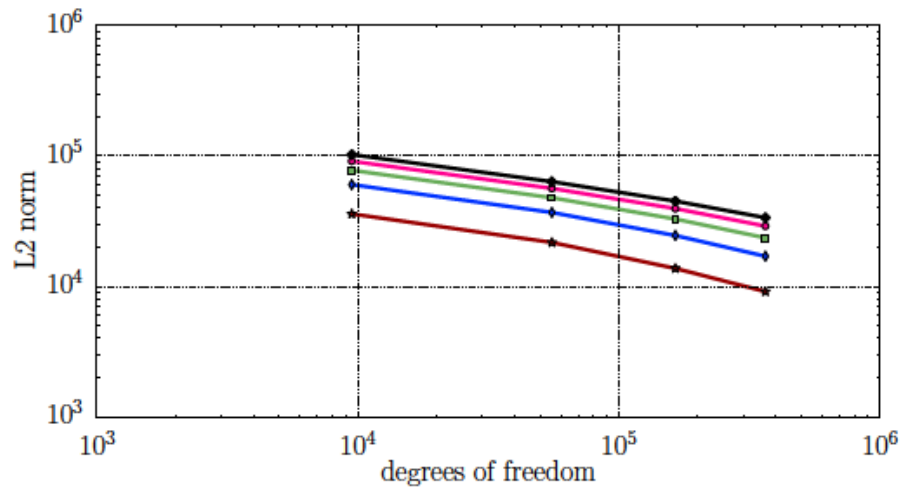

Fig. 5 For the linear elastic transient FEM, plot of degrees of freedom versus $L_{2}$ norm over the whole mesh in a log-log scale. Colors denote different time steps

\section{Appendix B. Convergence for nonlinear elasticity}

Standard $h$-convergence has been conducted with the same geometry and boundary conditions for the first five time steps, $t_{1}, t_{2}, t_{3}, t_{4}$, and $t_{5}$. The results are compiled in Table 3 and shown in Fig. 6.

Table 3 Convergence analysis for nonlinear elastic transient FEM computation

\begin{tabular}{llllll}
\hline dofs & $t_{1}$ & $t_{2}$ & $t_{3}$ & $t_{4}$ & $t_{5}$ \\
\cline { 2 - 5 } & $L_{2}$ norm & & & & \\
\hline 14553 & 38.564 & 76.962 & 115.198 & 153.275 & 191.195 \\
105903 & 40.346 & 80.586 & 120.727 & 160.774 & 200.733 \\
346053 & 40.755 & 81.440 & 122.066 & 162.641 & 203.177 \\
807003 & 40.920 & 81.791 & 122.628 & 163.446 & 204.259 \\
\hline
\end{tabular}

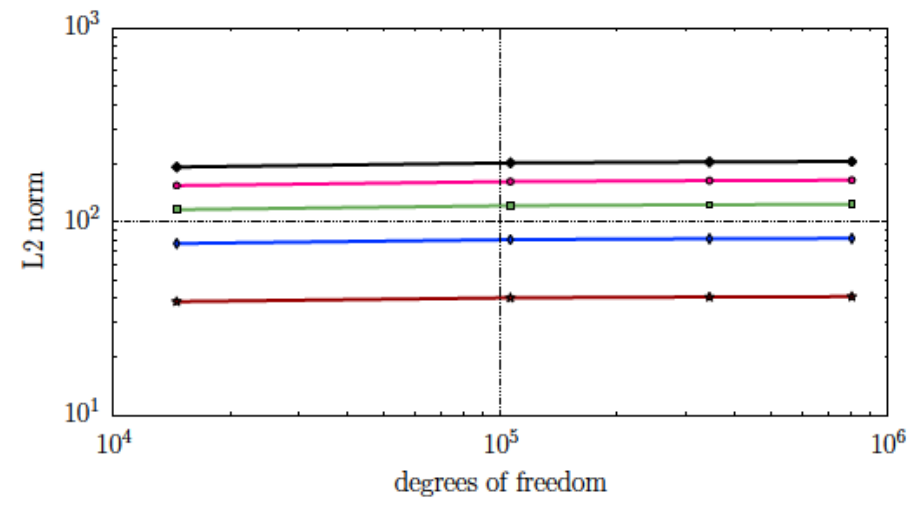

Fig. 6 For the nonlinear elastic transient FEM, plot of degrees of freedom versus $L_{2}$ norm over the whole mesh in a log-log scale. Colors denote different time steps

\section{Appendix C: Convergence for linear strain gradient elasticity}

Standard $h$-convergence has been conducted with the same geometry and boundary conditions for the first five time steps, $t_{1}, t_{2}, t_{3}, t_{4}$, and $t_{5}$. The results are compiled in Table 4 and shown in Fig. 7. 
Table 4 Convergence analysis for linear strain gradient elastic transient FEM computation

\begin{tabular}{llllll}
\hline dofs & $t_{1}$ & $t_{2}$ & $t_{3}$ & $t_{4}$ & $t_{5}$ \\
\cline { 2 - 5 } & $L_{2}$ norm & & & & \\
\hline 9075 & 0.005499 & 0.021587 & 0.058142 & 0.134536 & 0.288832 \\
26607 & 0.005520 & 0.021714 & 0.058642 & 0.136126 & 0.293113 \\
58563 & 0.005536 & 0.021813 & 0.059021 & 0.137123 & 0.295191 \\
109263 & 0.005549 & 0.021895 & 0.059326 & 0.138029 & 0.297284 \\
\hline
\end{tabular}

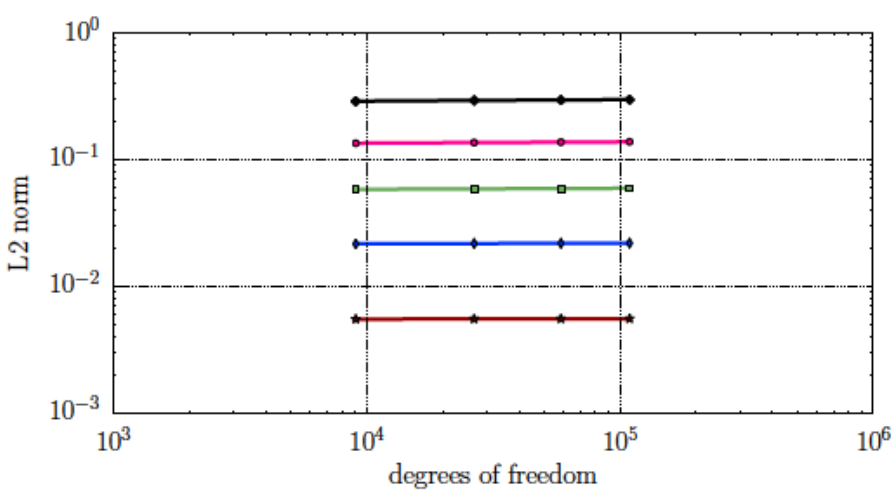

Fig. 7 For the linear strain gradient elastic transient FEM, plot of degrees of freedom versus $L_{2}$ norm over the whole mesh in a $\log -\log$ scale. Colors denote different time steps

\section{References}

1. Abali, B.E.: Computational Reality, Solving Nonlinear and Coupled Problems in Continuum Mechanics. Advanced Structured Materials. Springer Nature, Singapore (2017a)

2. Abali, B.E.: Technical University of Berlin, Institute of Mechanics, Chair of Continuums Mechanics and Material Theory.Computational Reality. http://www.lkm.tu-berlin.de/ComputationalReality/ (2017b)

3. Abali, B.E., Müller, W.H.: Numerical solution of generalized mechanics based on a variational formulation. In Oberwolfach Reports - Mechanics of Materials, European Mathematical Society Publishing House 17(1), pp. 9-12 (2016)

4. Abali, B.E., Müller, W.H., Eremeyev, V.A.: Strain gradient elasticity with geometric nonlinearities and its computational evaluation. Mech. Adv. Mater. Modern Process. 1, 4 (2015)

5. Ahrens, J., Geveci, B., Law, C.: ParaView: An End-User Tool for Large Data Visualization. In: Visualization Handbook, chapter 36. Elsevier Academic Press (2011)

6. Alnæs, M.S., Mardal, K.-A.: On the efficiency of symbolic computations combined with code generation for finite element methods. ACM Trans. Math. Softw. (TOMS) 37(1), 6 (2010)

7. Alnaes, M.S., Mardal, K.A.: SyFi and SFC: Symbolic Finite Elements and Form Compilation, vol. 15. Springer, Berlin (2012)

8. Auffray, N., dell'Isola, F., Eremeyev, V., Madeo, A., Rosi, G.: Analytical continuum mechanics à la Hamilton-Piola least action principle for second gradient continua and capillary fluids. Math. Mech. Solids 20(4), 375-417 (2015)

9. Ayachit, U.: The paraview guide: a parallel visualization application. Technical report, Kitware (2015)

10. Barenblatt, G.I.: Scaling, Self-similarity, and Intermediate Asymptotics: Dimensional Analysis and Intermediate Asymptotics. Cambridge University Press, Cambridge (1996)

11. Bilotta, A., Formica, G., Turco, E.: Performance of a high-continuity finite element in three-dimensional elasticity. Int. J. Numer. Methods Biomed. Eng. 26(9), 1155-1175 (2010)

12. Brezny, R., Green, D.: Characterization of edge effects in cellular materials. J. Mater. Sci. 25(11), 4571-4578 (1990)

13. Chen, C., Fleck, N.: Size effects in the constrained deformation of metallic foams. J. Mech. Phys. Solids 50(5), 955-977 (2002)

14. dell'Isola, F., Andreaus, U., Placidi, L.: At the origins and in the vanguard of peridynamics, non-local and higher-gradient continuum mechanics: an underestimated and still topical contribution of gabrio piola. Math. Mech. Solids 20, 887-928 (2014)

15. dell'Isola, F., Sciarra, G., Vidoli, S.: Generalized Hooke's law for isotropic second gradient materials. Proc. R. Soc. A Math. Phys. Eng. Sci. 465, 2177-2196 (2009)

16. Eremeyev, V.A.: On equilibrium of a second-gradient fluid near edges and corner points. In: Advanced Methods of Continuum Mechanics for Materials and Structures, pp. 547-556. Springer (2016)

17. Eremeyev, V.A., Altenbach, H.: Equilibrium of a second-gradient fluid and an elastic solid with surface stresses. Meccanica 49(11), 2635-2643 (2014)

18. Eringen, A.: Theory of micropolar elasticity. Technical report, DTIC Document (1967)

19. Giorgio, I.: Numerical identification procedure between a micro-cauchy model and a macro-second gradient model for planar pantographic structures. Zeitschrift für angewandte Mathematik und Physik 67(4), 95 (2016) 
20. Gnu Public . Gnu general public license. http://www.gnu.org/copyleft/gpl.html (2007)

21. Hoffman, J., Jansson, J., Johnson, C., Knepley, M., Kirby, R., Logg, A., Scott, L. R., Wells, G.N.: Fenics. (http://www. fenicsproject.org/) (2005)

22. Javili, A., dell'Isola, F., Steinmann, P.: Geometrically nonlinear higher-gradient elasticity with energetic boundaries. J. Mech. Phys. Solids 61(12), 2381-2401 (2013)

23. Jones, E., Oliphant, T., Peterson, P., et al.: SciPy: Open source scientific tools for Python. http://www.scipy.org/ (2001)

24. Kesler, O., Gibson, L.J.: Size effects in metallic foam core sandwich beams. Mater. Sci. Eng. A 326(2), 228-234 (2002)

25. Langanten, H.P., Logg, A.: Solving PDEs in Python, Volume 3 of Simula SpringerBriefs on Computing. Springer, Berlin (2017)

26. Logg, A., Mardal, K.A., Wells, G.N.: Automated Solution of Differential Equations by the Finite Element Method the FEniCS Book, Volume 84 of Lecture Notes in Computational Science and Engineering. Springer, Berlin (2011)

27. Mindlin, R.D.: Micro-structure in linear elasticity. Arch. Ration. Mech. Anal. 16(1), 51-78 (1964)

28. Mindlin, R.D.: Second gradient of strain and surface-tension in linear elasticity. Int. J. Solids Struct. 1(4), 417-438 (1965)

29. Mindlin, R.D., Tiersten, H.F.: Effects of couple-stresses in linear elasticity. Arch. Ration. Mech. Anal. 11, 415-448 (1962)

30. Morrison, J.: The yield of mild steel with particular reference to the effect of size of specimen. Proc. Inst. Mech. Eng. 142(1), 193-223 (1939)

31. Neff, P., Ghiba, I.-D., Madeo, A., Placidi, L., Rosi, G.: A unifying perspective: the relaxed linear micromorphic continuum. Contin. Mech. Thermodyn. 26(5), 639-681 (2014)

32. Oliphant, T.E.: Python for scientific computing. Comput. Sci. Eng. 9(3), 10-20 (2007)

33. Pideri, C., Seppecher, P.: A homogenization result for elastic material reinforced periodically with high rigidity elastic fibres. Comptes Rendus de l'Academie des Sci. Ser. IIB Mech. Phys. Chem. Astron. 8(324), 475-481 (1997)

34. Placidi, L., Andreaus, U., Della Corte, A., Lekszycki, T.: Gedanken experiments for the determination of two-dimensional linear second gradient elasticity coefficients. Zeitschrift für angewandte Mathematik und Physik 66(6), 3699-3725 (2015)

35. Polizzotto, C.: A second strain gradient elasticity theory with second velocity gradient inertia-part i: constitutive equations and quasi-static behavior. Int. J. Solids Struct. 50(24), 3749-3765 (2013a)

36. Polizzotto, C.: A second strain gradient elasticity theory with second velocity gradient inertia-part ii: dynamic behavior. Int. J. Solids Struct. 50(24), 3766-3777 (2013b)

37. Reiher, J.C., Giorgio, I., Bertram, A.: Finite-element analysis of polyhedra under point and line forces in second-strain gradient elasticity. J. Eng. Mech. 143, 04016112 (2016)

38. Romano, G., Barretta, R., Barretta, A.: On maupertuis principle in dynamics. Rep. Math. Phys. 63(3), 331-346 (2009)

39. Scerrato, D., Zhurba Eremeeva, I.A., Lekszycki, T., Rizzi, N.L.: On the effect of shear stiffness on the plane deformation of linear second gradient pantographic sheets. ZAMM J. Appl. Math. Mech./Zeitschrift für Angewandte Mathematik und Mechanik 96(11), 1268-1279 (2016)

40. Steigmann, D.J., dell'Isola, F.: Mechanical response of fabric sheets to three-dimensional bending, twisting, and stretching. Acta Mech. Sin. 31(3), 373-382 (2015)

41. Toupin, R.A.: Theories of elasticity with couple-stress. Arch. Ration. Mech. Anal. 17(2), 85-112 (1964)

42. Turco, E., Golaszewski, M., Cazzani, A., Rizzi, N.L.: Large deformations induced in planar pantographic sheets by loads applied on fibers: experimental validation of a discrete lagrangian model. Mech. Res. Commun. 76, 51-56 (2016)

43. Yang, J., Lakes, R.S.: Experimental study of micropolar and couple stress elasticity in compact bone in bending. J. Biomech. 15(2), 91-98 (1982) 